\title{
ASE Noise in Raman Amplifiers: Pump Depletion Impact
}

\author{
Samer DARWISH ${ }^{1}$, Ahmed SAMRA ${ }^{1}$, Moustafa ALY $Y^{2}$ \\ ${ }^{1}$ Dept. of Electronics and Communications, Faculty of Engineering, Mansoura University, Mansoura, Egypt \\ ${ }^{2}$ Electronics and Communications Engineering Dept., College of Engineering and Technology, \\ Arab Academy for Science, Technology and Maritime Transport, Alexandria, Egypt \\ samer_drawish@yahoo.com, ahsamra@yahoo.co.uk,drmosaly@gmail.com
}

Submitted July 23, 2017 / Accepted October 19, 2017

\begin{abstract}
This paper provides a detailed analysis for the effect of pump depletion on amplified spontaneous emission (ASE) noise power, optical signal to noise ratio (OSNR), and noise figure (NF) in forward pumped distributed Raman fiber amplifier (DRFA). The optimum pump power for high OSNR, low NF, and better gain is obtained $\sim 256 \mathrm{~mW}$ at input signal power of $10^{-6} \mathrm{~W}$, fiber length of $120 \mathrm{~km}$, fiber loss of $0.2 \mathrm{~dB} / \mathrm{km}$, and optical filter bandwidth of $0.5 \mathrm{~nm}$. The obtained results are compared with the previously published ones showing a good agreement.
\end{abstract}

\section{Keywords}

Pump depletion, optimum pump power, DRFA, ASE, OSNR, NF

\section{Introduction}

During the nonelastic collision between incident photon and a molecule of the material of the optical fiber, the incident photon transmits some part of energy to the molecule of the material or receives some energy from the molecule. Regarding to the fact, that energy of the photon corresponds with its frequency, transmission or acceptance of energy leads to changes in frequency and wavelength of the light. This phenomenon was proved experimentally by the Indian scientist C. V. Raman in 1928 after whom this effect took its name Raman scattering [1].

Raman fiber amplifiers (RFAs) are known as stable high power fiber light sources providing almost any wavelength in the near IR range. This is due to their broad gain spectrum, low noise characteristics, and wavelength versatility of stimulated Raman scattering (SRS). Therefore, RFAs are very attractive for a variety of applications, especially in the fields of telecommunications. There are two types of RFAs: discrete or lumped and distributed Raman amplifier (DRFA). If the amplifier is contained in a box at the transmitter or receiver end of the system, it is called a discrete Raman amplifier, while the DRFA utilizes the transmission optical fiber as an active medium.

Amplified spontaneous emission (ASE) noise is one of the major sources of noise in DRFAs which restricts the amplification of signal. If a signal is allowed to propagate along the fiber with no loss and with no amplification, then its signal to noise ratio at the receiver end would be equal to that of the input value and its noise figure will be equal to one. But, in practical situations, this is not possible. Furthermore, the optical noise power caused by distributed Raman amplification is smaller than that of conventional EDFAs [2].

In a previous work [3], we theoretically discussed and investigated forward SRS in a DRFA. This was carried out with consideration of the pump depletion due to the SRS process. The effect of pump depletion on the performance of bidirectional pump Raman amplifiers was clarified. The effect of pump depletion was studied in forward pump Raman amplifier on the signal power, pump power, unity gain length, and amplifier gain. Mathematical expressions were derived for threshold depletion length, threshold depletion pump power, and unity gain length in forward pump Raman amplifier.

In this paper, we investigate the impact of pump depletion on ASE power, OSNR, and NF in a forward DRFA. An analytical model is proposed, based on the solution of differential equations for forward traveling waves of the signal power and the pump power obtained by Mochizuki [4].

The remainder of the paper is organized as follows. In Sec. 2, the analytical model for spontaneous Raman scattering power, optical signal to noise ratio and noise figure is presented. The obtained results are displayed and discussed in Sec. 3. Section 4 is devoted for the main conclusions.

\section{Model and Analysis}

When the pump power propagates in the direction of the signal it is called co- or forward pumping scheme. In case of pump depletion, signal power and pump power can be expressed as [3], [4]

$$
P_{S}(z)=\frac{P_{S}(0) \exp \left[\frac{g_{\mathrm{R}} P_{\mathrm{p}}(0)}{\alpha A}(1-\exp (-\alpha \mathrm{z}))-\alpha \mathrm{z}\right]}{1+\frac{v_{\mathrm{p}} P_{\mathrm{s}}(0)}{v_{\mathrm{s}} P_{\mathrm{p}}(0)} \exp \left[\frac{g_{\mathrm{R}} P_{\mathrm{p}}(0)}{\alpha A}(1-\exp (-\alpha \mathrm{z}))\right]}
$$




$$
\begin{aligned}
& \text { and } \\
& P_{\mathrm{p}}(z)=\frac{P_{\mathrm{p}}(0) \exp (-\alpha \mathrm{z})}{1+\frac{v_{\mathrm{p}} P_{\mathrm{s}}(0)}{v_{\mathrm{s}} P_{\mathrm{p}}(0)} \exp \left[\frac{g_{\mathrm{R}} P_{\mathrm{p}}(0)}{\alpha A}(1-\exp (-\alpha \mathrm{z}))\right]}
\end{aligned}
$$

where $P_{\mathrm{p}}$ and $P_{\mathrm{s}}$ represent the pump and signal powers of waves at frequencies $v_{\mathrm{p}}$ and $v_{\mathrm{s}}$ and both are functions of the propagation distance $z . g_{\mathrm{R}}, \alpha$ and $A$ are, respectively, the gain coefficient, the fiber loss parameter and the fiber effective area. We assume that $\alpha_{\mathrm{s}}=\alpha_{\mathrm{p}}=\alpha$ because the signal and pump wavelengths will be held around the $1.55 \mu \mathrm{m}$ region, where fibers are characterized by extremely low loss.

At any point (propagation distance) $z$, the photon occupation number, $N_{\mathrm{s}}(z)$, of a given mode due to spontaneous emission, is given by [5]

$$
N_{\mathrm{s}}(z)=\int_{0}^{z} \frac{g_{\mathrm{R}} P_{\mathrm{p}}(\xi)}{A} \exp \left[\alpha(\xi-z)+\int_{\xi}^{z} \frac{g_{\mathrm{R}} P_{\mathrm{p}}(\eta)}{A} \mathrm{~d} \eta\right] \mathrm{d} \xi .
$$

The total Stokes power due to forward spontaneous Raman scattering is given by [6]

$$
\begin{aligned}
P_{\text {spontaneous }}(z)= & \frac{B_{\mathrm{o}} h c^{2}}{\left(\lambda_{\mathrm{s}}\right)^{3}} \int_{0}^{z} \frac{g_{\mathrm{R}} P_{\mathrm{p}}(\xi)}{A} \exp [\alpha(\xi-z)+ \\
& \left.\int_{\xi}^{z} \frac{g_{\mathrm{R}} P_{\mathrm{p}}(\eta)}{A} \mathrm{~d} \eta\right] \mathrm{d} \xi
\end{aligned}
$$

where $\lambda_{\mathrm{s}}$ is the signal wavelength, $B_{\mathrm{o}}$ is the bandwidth of the optical filter, $c$ is the free space speed of light and $h$ is Planck's constant.

This is often referred to as ASE noise because of its amplification by the distributed Raman gain. The reason for estimating noise properties of networks is to minimize the problem of noise generated in receiving systems [7].

The OSNR is defined as the ratio of total optical power of the signal to the amplified spontaneous emission noise power [8], [9]

$$
O S N R=\frac{P_{\mathrm{S}}(L)}{P_{\text {spontaneous }}(L)} .
$$

The noise figure, NF, is a measure of the fineness of the optical fiber system. The noise figure of Raman amplifier, $N F(z)$, at distance $z$ from the input is defined as [9], [10]

$$
N F(z)=\frac{(S N R)_{\text {in }}}{(S N R)_{\text {out }}} .
$$

A perfect amplifier would amplify the noise at its input along with the signal, maintaining the same signal to noise ratio at its input and output.

The electrical signal to noise ratio in (6) points to the electric power generated when the optical signal is converted into an electric current. In general, NF depends on several detector parameters that govern thermal noise asso- ciated with the detector. A simple expression for NF can be obtained by considering an ideal detector whose performance is limited by shot noise only. The electrical SNR of the input signal is then given by [9]

$$
(S N R)_{\text {in }}=\frac{\left(I_{\mathrm{d}}\right)^{2}}{\sigma_{\mathrm{s}}^{2}}=\frac{\left(R_{\mathrm{d}} P_{\mathrm{in}}\right)^{2}}{\sigma_{\mathrm{s}}^{2}}
$$

where $I_{\mathrm{d}}$ is the current and $R_{\mathrm{d}}=q / h v_{\mathrm{s}}$ is the responsivity of a detector with $100 \%$ quantum efficiency. The variance of shot noise over detector bandwidth $\Delta f$ can be written as $\sigma_{\mathrm{S}}^{2}=2 q R_{\mathrm{d}} P_{\text {in }} \Delta f$, resulting in [9]

$$
(S N R)_{\text {in }}=\frac{P_{\text {in }}}{2 h v_{\mathrm{s}} \Delta f}
$$

where $q$ is the electron charge.

The total variance of output current fluctuations can be written as [9]

$$
\sigma_{\text {out }}^{2}=\sigma_{\mathrm{b}}^{2}+\sigma_{\mathrm{ASE}}^{2}+G_{\mathrm{L}} \sigma_{\mathrm{s}}^{2}+\sigma_{\mathrm{T}}^{2}
$$

where $G_{\mathrm{L}}$ is a gain of forward pumped distributed Raman fiber amplifier of length $L$ when pump depletion is considered. It can be obtained as [3]

$$
G_{\mathrm{L}}=\frac{\exp \left[\frac{g_{\mathrm{R}} P_{\mathrm{p}}(0)}{\alpha A}(1-\exp (-\alpha \mathrm{L}))-\alpha \mathrm{L}\right]}{1+\frac{v_{\mathrm{p}} P_{\mathrm{s}}(0)}{v_{\mathrm{s}} P_{\mathrm{p}}(0)} \exp \left[\frac{g_{\mathrm{R}} P_{\mathrm{p}}(0)}{\alpha A}(1-\exp (-\alpha \mathrm{L}))\right]}
$$

$\sigma_{\mathrm{b}}^{2}, \sigma_{\mathrm{ASE}}^{2}$, and $\sigma_{\mathrm{T}}^{2}$ represent variances of three noise terms fluctuating with time, resulting from signal-ASE, ASEASE beating, and thermal effects, respectively. More details about these terms are given in Appendix.

We can neglect the thermal noise contribution $\sigma_{\mathrm{T}}{ }^{2}$ as it is relatively small. The $\sigma_{\text {ASE }}^{2}$ term is also small in comparison with $\sigma_{\mathrm{b}}{ }^{2}$. For this reason, the electrical SNR of the amplified signal could be approximately written as [9]

$$
(S N R)_{\text {out }}=\frac{\left(I_{\mathrm{d}}\right)^{2}}{\sigma_{\mathrm{s}}^{2}}=\frac{\left(R_{\mathrm{d}} G_{\mathrm{L}} P_{\text {in }}\right)^{2}}{G_{\mathrm{L}} \sigma_{\mathrm{s}}^{2}+\sigma_{\mathrm{b}}^{2}} .
$$

Now, $N F(z)$ can be obtained as

$$
N F(z)=\frac{1}{G_{\mathrm{L}}}\left(1+\frac{2 S_{\mathrm{ASE}}}{h v_{\mathrm{s}}}\right)
$$

where [9]

$$
S_{\mathrm{ASE}}=\frac{p_{\text {spontaneous }}}{2 B_{\mathrm{o}}} \text {. }
$$

A detailed proof for (12) is given in Appendix.

\section{Results and Discussion}

The numerical values used to perform calculations are extracted from [3] as: pump wavelength $\lambda_{\mathrm{p}}=1.54 \mu \mathrm{m}$, signal wavelength $\lambda_{\mathrm{s}}=1.55 \mu \mathrm{m}, g_{\mathrm{R}}=3.8 \times 10^{-14} \mathrm{~m} / \mathrm{W}, B_{\mathrm{o}}=$ 
$0.5 \mathrm{~nm}, A=1.96 \times 10^{-11} \mathrm{~m}^{2}$, and $\alpha=\alpha_{\mathrm{s}}=\alpha_{\mathrm{p}}=0.2 \mathrm{~dB} / \mathrm{km}$. Simulation results through this work are obtained using MATHCAD (ver. 7).

\subsection{Pump Depletion Impact on ASE Power}

The ASE noise is an important obstacle in the path of optical communication which not only weakens the main signal but also badly affects the other specifications of DRFAs. Based on the described model in Sec. 2, the calculated ASE power versus fiber length at pump power of $0.3 \mathrm{~W}$ and $0.5 \mathrm{~W}$ is displayed in Fig. 1. As shown, as the fiber length increases, the ASE power increases until $P_{\mathrm{ASE}}(\max )=0.1 \mathrm{~mW}(-10 \mathrm{dBm})$ at $0.3 \mathrm{~W}$ pump power, $P_{\mathrm{ASE}}(\max )=10 \mathrm{~mW}(10 \mathrm{dBm})$ and $251 \mathrm{~mW}(24 \mathrm{dBm})$ at $0.5 \mathrm{~W}$ pump power when pump depletion is considered and when it is neglected, respectively, at $P_{\mathrm{s}}(0)=10^{-7} \mathrm{~W}$.

Figure 1 clearly shows that ASE power increases up to a certain fiber length, and then begins to decrease. This occurs because of insufficient forward pump power and getting high losses. This result is in a fair agreement with that obtained by Premaratne [11]. It is important to note that the effect of pump depletion appears in case of $0.5 \mathrm{~W}$ pump power, Fig. 1. This is expected because the effect of pump depletion appears after a certain value of pump power $P_{\mathrm{p}}(0)=330 \mathrm{~mW}$, which is called threshold depletion pump power [3].

Figure 2 illustrates the dependence of the ASE power on the pump power for fiber length $L=120 \mathrm{~km}$. It is clear that, as pump power increases, the ASE power increases. This is because the pump power increases not only the stimulated scattering but also the spontaneous emission. The gain will be saturated by increasing pump power that leads the ASE power to reach to a maximum level. This level is limited by the photon occupation number of a given mode due to spontaneous emission, $N_{\mathrm{s}}(z)$. The ASE noise power remains constant at $-3.87 \mathrm{dBm}$ after $P_{\mathrm{p}}(0)=0.33 \mathrm{~W}$ due to pump depletion which is logic and coincides with that obtained by S. Darwish et al. [3].

Figure 3 displays the variation of normalized ASE power with fiber length. Clearly, it can be seen that norma-

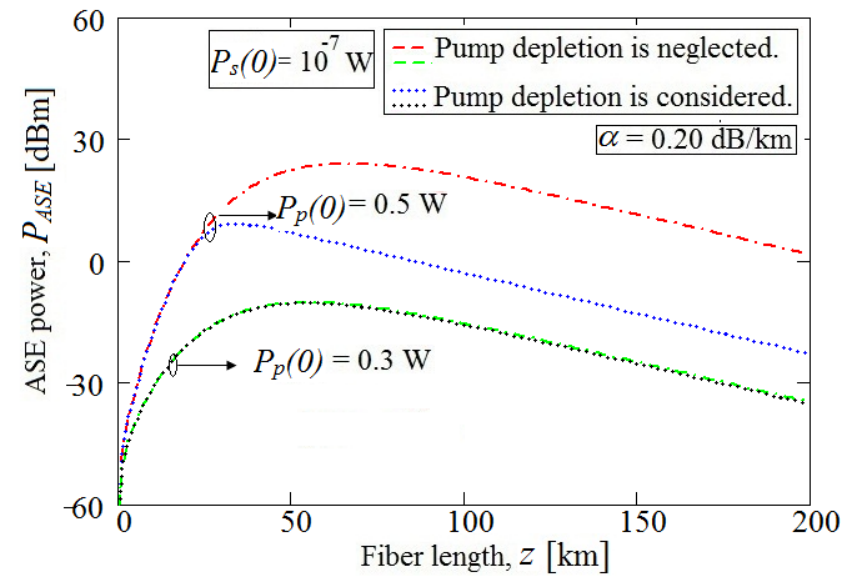

Fig. 1. ASE power as a function of fiber length $P_{\mathrm{p}}(0)=0.3 \mathrm{~W}$ and $P_{\mathrm{p}}(0)=0.5 \mathrm{~W}$.

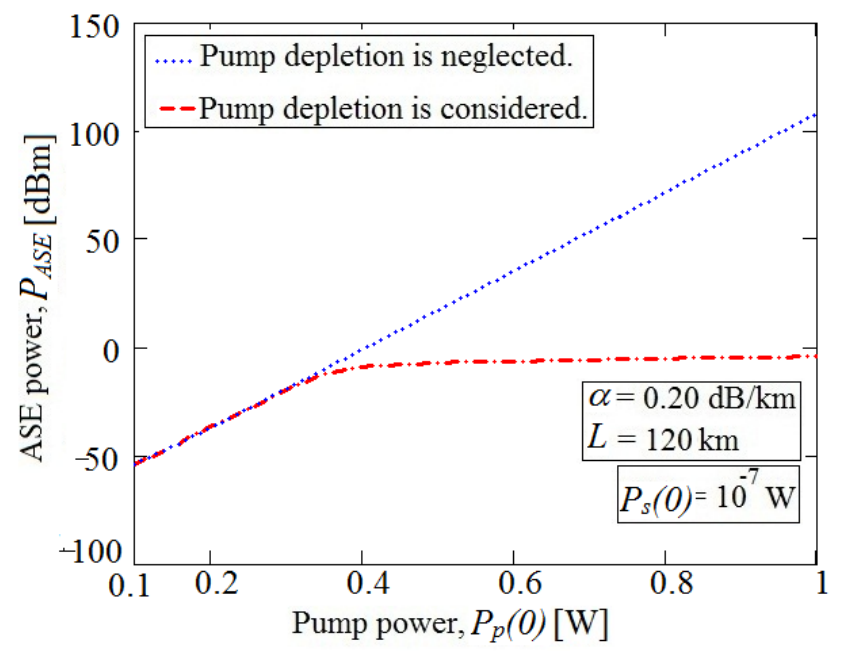

Fig. 2. ASE power against input pump power.

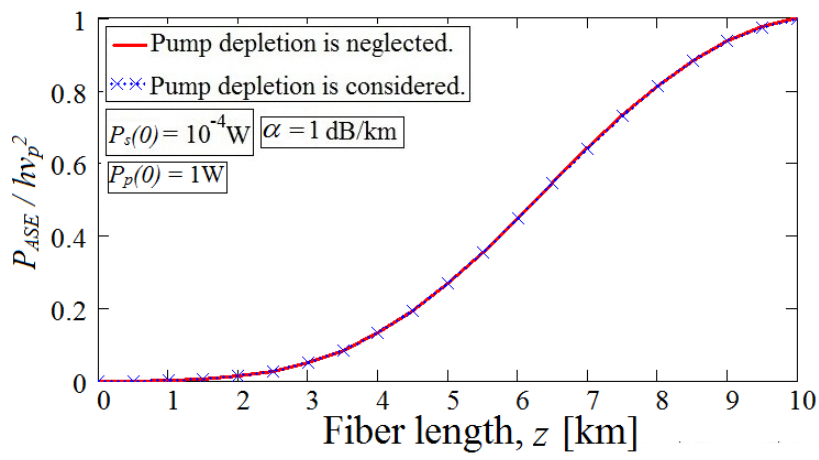

Fig. 3. ASE power normalized to $\left[1 / h v_{\mathrm{p}}{ }^{2}\right]$ as a function of fiber length.

lized ASE power increases with the fiber length when pump depletion is considered and when it is neglected. It is assumed that the fiber length up to $10 \mathrm{~km}$ is sufficiently short so that would not cause depletion for any frequency. We confirm the validity of the obtained results that are in a good agreement with the numerical results obtained by M. Dakss and P. Melman [7].

\subsection{Pump Depletion Impact on OSNR}

Figure 4 displays the dependence of the optical signal to noise ratio, OSNR, on pump power at different values of input signal power; $P_{\mathrm{s}}(0)=10^{-5}, 10^{-6}$, and $10^{-7} \mathrm{~W}$. As shown, as the input pump power increases, the OSNR decreases until $P_{\mathrm{p}}(0)=32 \mathrm{~mW}$ for $\alpha=0.2 \mathrm{~dB} / \mathrm{km}$. This is because the pump power over this range is weak and is not capable to compensate for attenuations in the signal as it propagates through the fiber. Thus, the signal power continues to decrease when the pump power is very low which results in decreasing OSNR. As pump power increases more than $32 \mathrm{~mW}$, the OSNR gradually increases when pump depletion is considered as shown in Fig. 4(a) and when pump depletion is neglected in Fig. 4(b). This increases the OSNR up to $28.75,19.22$, and $9.84 \mathrm{~dB}$, for $P_{\mathrm{s}}(0)=10^{-5}, 10^{-6}$, and $10^{-7} \mathrm{~W}$, respectively, because the pump power is sufficient for stimulated Raman scattering. 


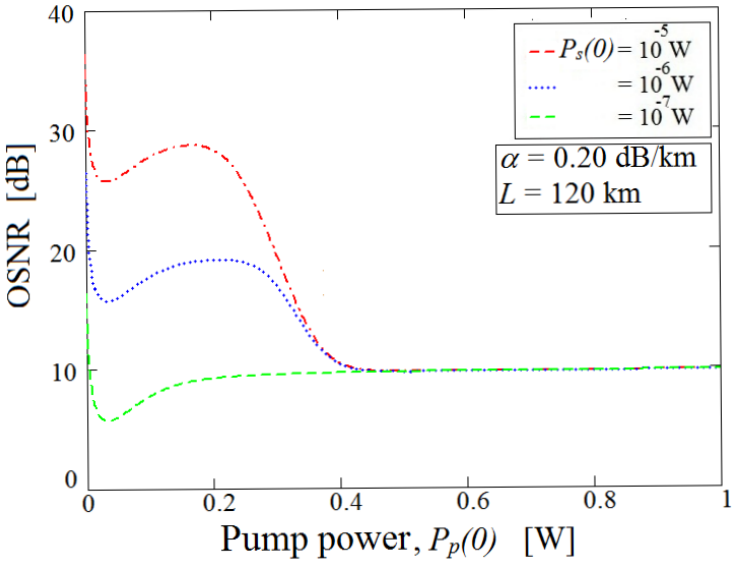

(a)

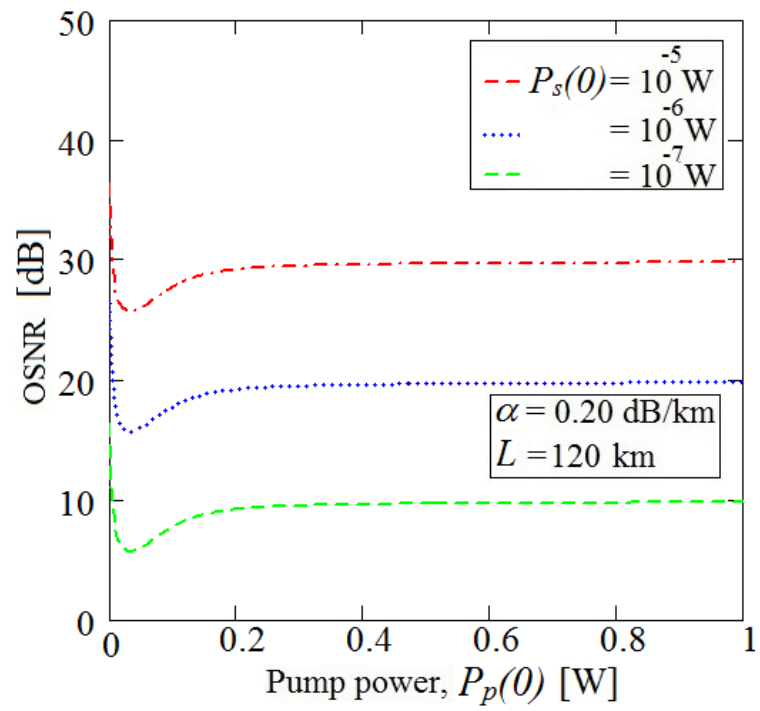

(b)

Fig. 4. Optical signal to noise ratio as a function of pump power at different values of input signal power: a) pump depletion is considered and b) pump depletion is ignored.

The OSNR starts to decrease to a saturation value after a certain value of $P_{\mathrm{p}}(0)=184$ and $235 \mathrm{~mW}$ at $P_{\mathrm{s}}(0)=$ $10^{-5}$ and $10^{-6} \mathrm{~W}$, respectively, due to pump depletion, as shown in Fig. 4(a). The OSNR will be almost constant after $P_{\mathrm{p}}(0)=250 \mathrm{~mW}$ for $P_{\mathrm{s}}(0)=10^{-7} \mathrm{~W}$ because the signal power is affected more by pump depletion at higher values of signal power [12], Fig. 4(a). As a comparison between Fig. 4(a) and Fig. 4(b), it can be seen that OSNR is reduced by $\sim 20$ and $10 \mathrm{~dB}$ at $P_{\mathrm{s}}(0)=10^{-5}$ and $10^{-6} \mathrm{~W}$, respectively, and fiber length $L=120 \mathrm{~km}$ in range of input pump power more than $450 \mathrm{~mW}$ due to pump depletion.

Figure 5 describes the effect of pump depletion on OSNR at different values of fiber loss. OSNR reduces for pump powers less than 40,36, and $30 \mathrm{~mW}$, at fiber loss $\alpha$ of $0.25,0.20$ and $0.15 \mathrm{~dB} / \mathrm{km}$, respectively. As pump power increases greater than 40,36 , and $30 \mathrm{~mW}$ at $\alpha=$ $0.25,0.20$ and $0.15 \mathrm{~dB} / \mathrm{km}$, respectively, the OSNR increases when pump depletion is considered or ignored. To state the issue clearly, after a threshold value of pump power 262, 235, and $158 \mathrm{~mW}$ for $\alpha=0.25,0.20$ and $0.15 \mathrm{~dB} / \mathrm{km}$, respectively, the pump depletion impact is seen

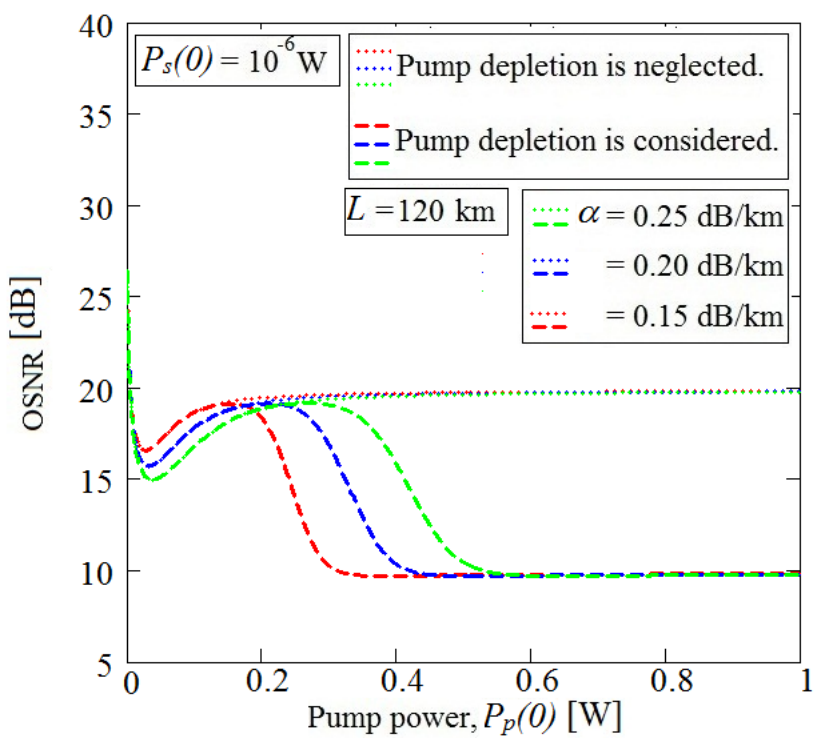

Fig. 5. Optical signal to noise ratio against input pump power at different values of fiber loss $\alpha$.

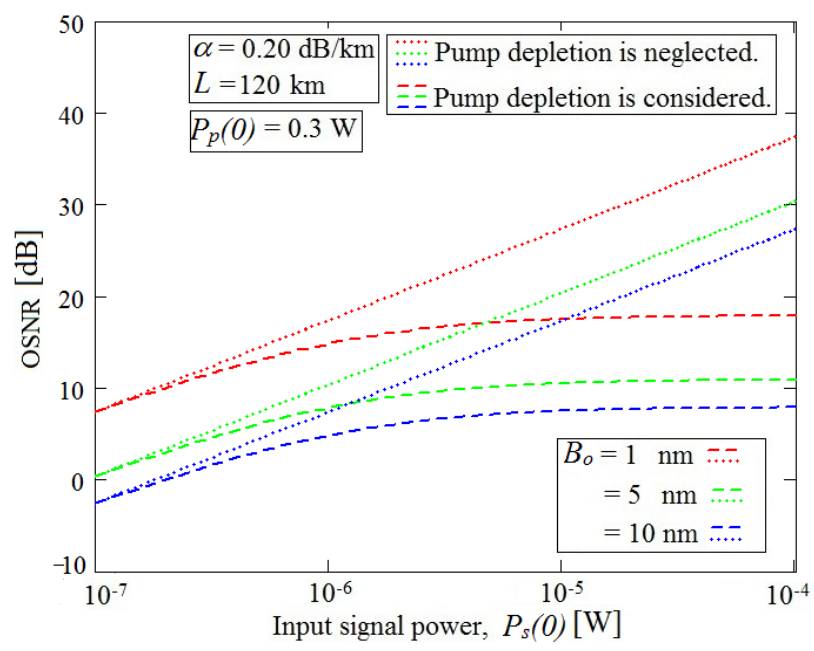

Fig. 6. Optical signal to noise ratio as a function of input signal power at different values of the optical filter bandwidth.

to degrade greatly the obtainable OSNR. These results coincide with our previous work [3]. After that, saturation values of OSNR are reached. The OSNR becomes almost constant at 9.8457 and $19.8175 \mathrm{~dB}$, for both cases when pump depletion is considered or ignored, respectively.

From Fig. 5, one can observe that the OSNR increases up to a saturation level. It can be inferred from Figs. 4 and 5 that the saturated value of OSNR is independent on input signal power in case when pump depletion is considered. This is because the value of input signal power has no role for high pump power as indicated in [3]. But, the fiber loss has no effect on the saturation value of OSNR in both cases when pump depletion is considered or ignored. The main reason is that the fiber loss degrades not only the signal power, but also the spontaneous emission.

Figure 6 shows the OSNR as a function of the input signal power for different optical filter bandwidths, at pump power $P_{\mathrm{p}}(0)=0.3 \mathrm{~W}$, fiber loss $\alpha=0.2 \mathrm{~dB} / \mathrm{km}$, and 
fiber length $L=120 \mathrm{~km}$. This is obtained for three different values of optical filter bandwidth; $B_{\mathrm{o}}=1,5$, and $10 \mathrm{~nm}$ by applying a signal input power from $10^{-7}$ to $10^{-4} \mathrm{~W}$ with and without pump depletion.

It is noted that OSNR increases significantly with increasing input signal power and decreasing optical filter bandwidth. As expected, the ASE power will decrease for small value of optical fiber bandwidth. The results displayed in Fig. 6 are in a good agreement with that reported in [6] when pump depletion is ignored. Also, when the optical bandwidth decreases and the input signal power increases, the saturation level of OSNR increases in case of pump depletion is considered. This is because the pump depletion is more effective for higher values of signal power [3].

\subsection{Pump Depletion Impact on NF}

Since the receiver will convert all photons to electrons, spontaneous emission exiting the optical amplifier will give rise to noise in the electrical domain. Figure 7 displays noise figure and the gain with and without pump depletion effect by tuning the input pump power from 0 to $1 \mathrm{~W}$. For fiber length $L=200 \mathrm{~km}$ and input signal power $P_{\mathrm{s}}(0)=10^{-6} \mathrm{~W}$, the noise figure decreases with the pump power and then goes to saturation $(\sim 0.177 \mathrm{~dB})$ after a certain level of pump power; $P_{\mathrm{p}}(0)=330 \mathrm{~mW}$, in both cases: when pump depletion is considered and when it is ignored.

Obviously, the gain increases with the increasing pump power. After a certain value of pump power, the gain becomes almost constant due to pump depletion in the stimulated process. This is in a good agreement with results reported in [3]. Clearly, the NF increases in case when pump depletion is considered. This is expected because the pump depletion reduces the amplifier gain [3] and NF varies inversely with the amplifier gain. The same behavior of NF versus pump power is obtained by Dimitropoulos et al. [13]. The high gain causes the spontaneous emission to stay in low levels. The NF of the DRFA varies linearly with ASE power and inversely with the amplifier gain. Therefore, the NF of the DRFA could be reduced to a minimum level by increasing the amplifier gain.

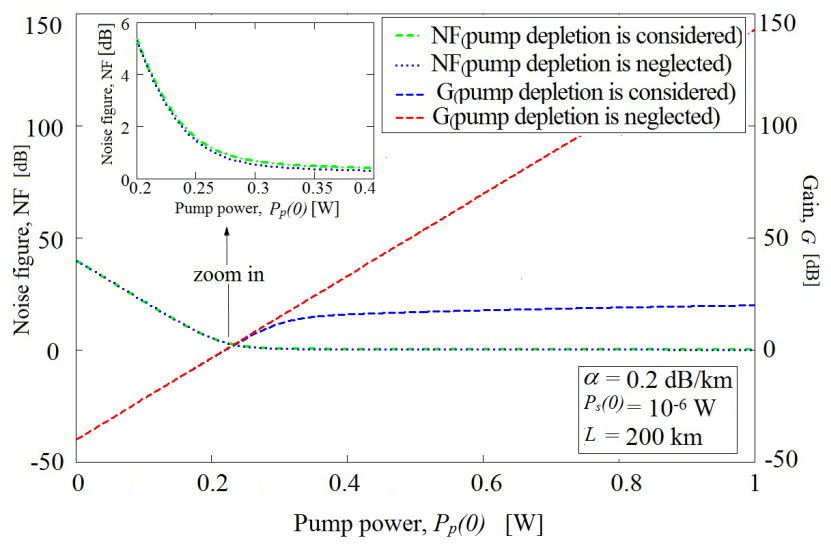

Fig. 7. Noise figure and amplifier gain as a function of input pump power with and without pump depletion.

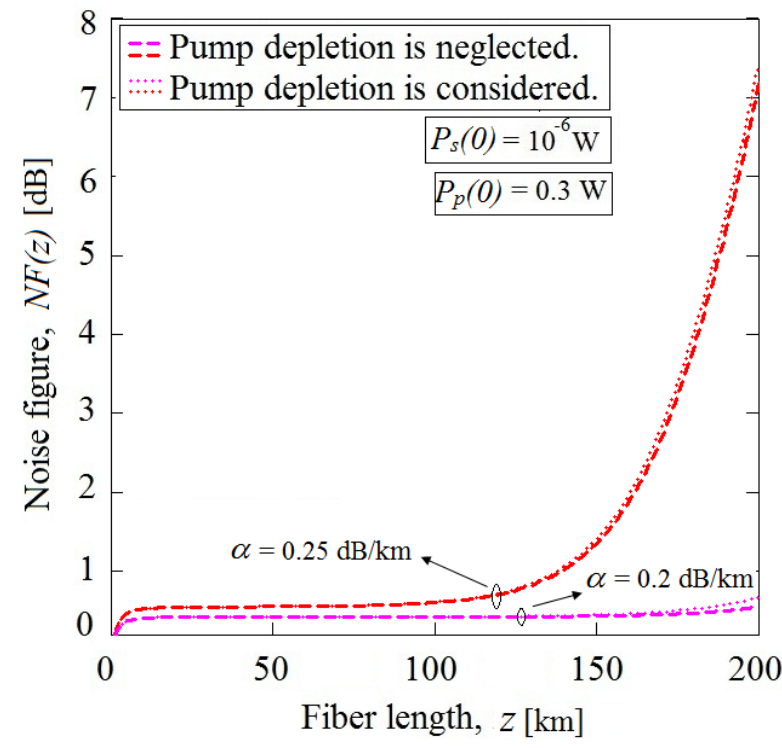

Fig. 8. Noise figure against fiber length for cases when pump depletion is considered and when it is ignored at $\alpha=0.25$ and $0.20 \mathrm{~dB} / \mathrm{km}$.

$\mathrm{NF}$ is a useful parameter to measure the degradation of SNR along the fiber length. It is plotted using (12) in Fig. 8. Both cases of pump depletion are considered. The values of different parameters used are $P_{\mathrm{s}}(0)=10^{-6} \mathrm{~W}$ and $P_{\mathrm{p}}(0)=300 \mathrm{~mW}$, for two different loss coefficient $\alpha=0.2$ and $0.25 \mathrm{~dB} / \mathrm{km}$. Figure 8 shows that NF increases with the increase of the fiber length. Also, as the fiber loss coefficient increases, NF increases because the small value of amplifier gain leads to high NF. For a loss coefficient of $0.25 \mathrm{~dB} / \mathrm{km}$, an increase in NF after $100 \mathrm{~km}$ is clearly noticed. This occurs because of the gain decrease with sharp pump depletion. This result has a fair agreement with that obtained by Isoe et al. [14].

\section{Conclusion}

We discussed the effect of pump depletion on the ASE power, OSNR, and NF for forward pumped DFRAs. The obtained results reveal that the effect of pump depletion appears after a threshold value of pump power: 330 , 235 , and $184 \mathrm{~mW}$ at input signal power of $10^{-7}, 10^{-6}$ and $10^{-5} \mathrm{~W}$, respectively, for fiber length $L=120 \mathrm{~km}, B_{\mathrm{o}}=$ $0.5 \mathrm{~nm}$ and $\alpha=0.2 \mathrm{~dB} / \mathrm{km}$. Although the pump depletion reduces the ASE power, the OSNR decreases because the signal power is more affected by pump depletion than the ASE power. Hence, the OSNR reaches its maximum value of 29.66 and $20.11 \mathrm{~dB}$ at $P_{\mathrm{p}}(0)$ of 160 and $200 \mathrm{~mW}$, respectively. Both values of pump power are below the threshold value to avoid pump depletion for $P_{\mathrm{s}}(0)=10^{-5}$ and $10^{-6} \mathrm{~W}$, respectively. Furthermore, limiting the optical filter bandwidth leads to achieve a high saturation level of OSNR when pump depletion is present. Accordingly, the dump depletion has a very small effect on the NF because both amplifier gain and ASE power decrease by pump depletion. It can also be concluded that the optimum pump power for high OSNR, low NF, and better gain is obtained $(\sim 256 \mathrm{~mW})$ at input signal power of $10^{-6} \mathrm{~W}$, fiber length of 
$120 \mathrm{~km}$, fiber loss of $0.2 \mathrm{~dB} / \mathrm{km}$, and optical filter bandwidth of $0.5 \mathrm{~nm}$. The preceding discussion does not mean that pump depletion is harmful in the amplification process, though, it is essential to be considered.

\section{References}

[1] KoudelKa, P., Petrujova, B., LATAL, J., et al. Optical fiber distributed sensing system applied in cement concrete commixture research. Radioengineering, 2010, vol. 19, no. 1, p. $172-177$. ISSN: $1805-9600$

[2] TAKACHIO, N., SUZUKI, H. Application of Raman-distributed amplification to WDM transmission systems using $1.55 \mu \mathrm{m}$ dispersion shifted fiber. Journal of Lightwave Technology, 2001, vol. 19 , no. 1, p. 60-69. DOI: 10.1109/50.914486

[3] DARWISH, S., SAMRA, A., MAHMOUD, M., ALY M. H. Pump depletion impact in fiber Raman amplifier. Journal of Optoelectronics and Advanced Materials, 2016, vol. 18, p. 421-427. ISSN: $1454-4164$

[4] MOCHIZUKI, K. Optical fiber transmission systems using stimulated Raman scattering theory. Journal of Lightwave Technology, 1985, vol. 3, no. 3, p. 688-694. DOI: 10.1109/JLT.1985.1074233

[5] SMITH, R. G. Optical power handling capacity of low loss optical fibers as determined by stimulated Raman and Brillouin scattering. Applied Optics, 1972, vol. 11, p. 2489-2494. DOI: 10.1364/AO.11.002489

[6] MOCHIZUKI, K., EDAGAWA, N., IWAMOTO, Y. Amplified spontaneous Raman scattering in fiber Raman amplifiers. Journal of Lightwave Technology, 1986, vol. 4, no. 9, p. 1328-1333. DOI: 10.1109/JLT.1986.1074905

[7] DAKSS, M. L., MELMAN, P. Amplified spontaneous Raman scattering and gain in fiber Raman amplifiers. Journal of Lightwave Technology, 1985, vol. 3, no. 4, p. 806-813. DOI: 10.1109/JLT.1985.1074270

[8] MOENCH, W., LOECKLIN, E. Measurement of optical signal to noise ratio in coherent systems using polarization multiplexed transmission. In Proceedings of Optical Fiber Communication Conference (OFC 2017). Los Angeles (USA), 2017, p. Th2A.42. ISBN: 978-1-943580-23-1. DOI: 10.1364/OFC.2017.Th2A.42

[9] HEAdley, C., AGRAWAL, G. P. Raman Amplifier in Fiber Optical Communication Systems. 1st ed. Burlington (USA): Elsevier Academic Press, 2005. ISBN: 0-12-044506-9

[10] FRIIS, S., MEJLING, L., ROTTWITT, K. Effects of Raman scattering and attenuation in silica fiber based parametric frequency conversion. Optics Express, 2017, vol. 25, no. 7, p. 7324-7337. DOI: 10.1364/OE.25.007324

[11] PREMARATNE, M. Analytical characterization of optical power and noise figure of forward pumped Raman amplifiers. Optics Express, 2004, vol. 12, no. 18, p. 4235-4245. DOI: 10.1364/OPEX.12.004235

[12] BROMAGE, J. Raman amplification for fiber communications systems. Journal of Lightwave Technology, 2004, vol. 22, no. 1, p. 79-93. DOI: $10.1109 /$ JLT.2003.822828

[13] DIMITROPOULOS, D., SOLLI, D. R., CLAPS, R., JALALI, B. Noise figure and photon statistics in coherent anti-Stokes Raman scattering. Optics Express, 2006, vol. 14, no. 23, p. 11418-11432. DOI: 10.1364/OE.14.011418

[14] ISOE, G. M., MUGURO, K. M., WASWA, D. W. Noise figure analysis of distributed fibre Raman amplifier. International Journal of Scientific \& Technology Research, 2013, vol. 2, no. 11, p. 375-378. ISSN: $2277-8616$

\section{Appendix A}

The noise figure is defined as [9], [10]

$$
N F(z)=\frac{(S N R)_{\text {in }}}{(S N R)_{\text {out }}} .
$$

The input signal to noise ratio is given as [9]

$$
(S N R)_{\text {in }}=\frac{\left(I_{\mathrm{d}}\right)^{2}}{\sigma_{\mathrm{s}}^{2}}
$$

where

$$
\begin{aligned}
& I_{\mathrm{d}}=R_{\mathrm{d}} P_{\text {in }}, \\
& R_{\mathrm{d}}=\frac{q}{h v_{\mathrm{s}}},
\end{aligned}
$$

$h$ is Planck's constant, and

$$
\sigma_{\mathrm{S}}^{2}=2 q R_{\mathrm{d}} P_{\text {in }} \Delta f .
$$

Hence, (A2) becomes

$$
(S N R)_{\text {in }}=\frac{P_{\text {in }}}{2 h v_{\mathrm{s}} \Delta f} .
$$

The output signal to noise ratio can be obtained as [9]

$$
(S N R)_{\text {out }}=\frac{\left(I_{\mathrm{d}}\right)^{2}}{\sigma_{\text {noise }}^{2}} .
$$

Signal detector current can be written as [9]

$$
I_{\mathrm{d}}=R_{\mathrm{d}} \mathrm{G}_{\mathrm{L}} P_{\text {in }} .
$$

When all noise sources are included, the detector current takes the form [9]

$$
I_{\mathrm{d}}=R_{\mathrm{d}} G_{\mathrm{L}} P_{\text {in }}+i_{\mathrm{b}}+i_{\mathrm{ASE}}+i_{\mathrm{s}}+i_{\mathrm{T}}
$$

where $i_{\mathrm{b}}, i_{\mathrm{ASE}}, i_{\mathrm{s}}$, and $i_{\mathrm{T}}$ represent current fluctuations resulting from signal-ASE, ASE-ASE beating, shot noise, and thermal noise, respectively.

The total variance of current fluctuations can be written from (A9) as [9]

$$
\sigma_{\text {noise }}^{2}=\sigma_{\mathrm{b}}^{2}+\sigma_{\mathrm{ASE}}^{2}+G_{\mathrm{L}} \sigma_{\mathrm{s}}^{2}+\sigma_{\mathrm{T}}^{2}
$$

where [7]

$$
\begin{gathered}
\sigma_{\mathrm{b}}^{2}=4 R_{\mathrm{d}}^{2} G_{\mathrm{L}} P_{\mathrm{in}} S_{\mathrm{ASE}} \Delta f, \\
\sigma_{\mathrm{ASE}}^{2}=4 R_{\mathrm{d}}^{2} S_{\mathrm{ASE}}^{2} \Delta f\left(B_{\mathrm{o}}-\frac{\Delta f}{2}\right), \\
\sigma_{\mathrm{s}}^{2}=2 q R_{\mathrm{d}} P_{\text {in }} \Delta f .
\end{gathered}
$$

We can neglect the thermal noise contribution $\sigma_{\mathrm{T}}^{2}$ as it is relatively small. The $\sigma_{\mathrm{ASE}}{ }^{2}$ term is also small in comparison with $\sigma_{\mathrm{b}}{ }^{2}$. Equation (A10) becomes

$$
\sigma_{\text {noise }}^{2}=\sigma_{\mathrm{b}}^{2}+G_{\mathrm{L}} \sigma_{\mathrm{s}}^{2} \text {. }
$$

For this reason, the electrical SNR of the amplified signal is approximately given by [9] 


$$
\begin{aligned}
(S N R)_{\text {out }}= & \frac{\left(R_{\mathrm{d}} \mathrm{G}_{\mathrm{L}} P_{\text {in }}\right)^{2}}{4 R_{\mathrm{d}}^{2} G_{\mathrm{L}} P_{\text {in }} S_{\text {ASE }} \Delta f+G_{\mathrm{L}} 2 q R_{\mathrm{d}} P_{\text {in }} \Delta f}= \\
& \frac{R_{\mathrm{d}} G_{\mathrm{L}} P_{\text {in }}}{2 q \Delta f+4 R_{\mathrm{d}} S_{\mathrm{ASE}} \Delta f} .
\end{aligned}
$$

Substituting (A6) and (A15) into (A1), one can get

$$
\begin{gathered}
N F(z)=\frac{2 G_{\mathrm{L}} q R_{\mathrm{d}} P_{\mathrm{in}} \Delta f+4 R_{\mathrm{d}}^{2} G_{\mathrm{L}} P_{\mathrm{in}} S_{\mathrm{ASE}} \Delta f}{2 h v_{\mathrm{s}} \Delta f R_{\mathrm{d}}^{2} G_{\mathrm{L}}^{2} P_{\mathrm{in}}}= \\
\frac{q+2 R_{\mathrm{d}} S_{\mathrm{ASE}}}{h v_{\mathrm{s}} R_{\mathrm{d}} G_{\mathrm{L}}}=\frac{1}{G_{\mathrm{L}}}\left(1+\frac{2 S_{\mathrm{ASE}}}{h v_{\mathrm{s}}}\right) .
\end{gathered}
$$

\section{About the Authors...}

Samer DARWISH was born in Alexandria, Egypt in 1974. He received his B.Sc. degree in Communications from the Faculty of Engineering, Alexandria University, Egypt, in 1996. He received his M.Sc. degree in Optical Amplifiers from the College of Engineering and Technology, Arab Academy for Science, Technology and Maritime Transport (AASTMT) in 2006. His research interests include optical amplifiers and optical fiber communications.

Ahmed S. SAMRA was born in 1954, Mansoura, Egypt.
He received his B.Sc. and M.Sc. from the Faculty of Electronic Engineering, Menoufia University, Egypt, in 1977 and 1982, respectively. He received his Ph.D. degree in Optical Communications and Integrated Optics from ENSEG, Grenoble, France in 1988. He is a professor of Optical Communications, Electronics and Communications Engineering Dept., Faculty of Engineering, Mansoura University, Mansoura, Egypt. He is the director of Biomedical Engineering Program, Faculty of Engineering, Mansoura University, Egypt. He was a co-supervisor of 60 M.Sc. and $\mathrm{Ph}$.D. students. He is a reviewer in some international journals (Optical Engineering, Optik, Optoelectronics and Advanced Materials Rapid Communications). His research area includes optical communications, integrated optics and optical measurement techniques.

Moustafa H. ALY (corresponding author) was born in 1953, Alexandria, Egypt. He received his B.Sc., M.Sc. and $\mathrm{Ph}$.D. from the Faculty of Engineering, Alexandria University, Alexandria, Egypt, respectively in 1976, 1983 and 1987. He is a professor of Optical Communications, Electronics and Communications Engineering Dept., College of Engineering and Technology, Arab Academy for Science, Technology and Maritime Transport, Alexandria, Egypt. He was a co-supervisor of 110 M.Sc. and Ph.D. students and published 220 journal and conference papers. His research area includes optical communications, optical amplifiers and optical networks. 